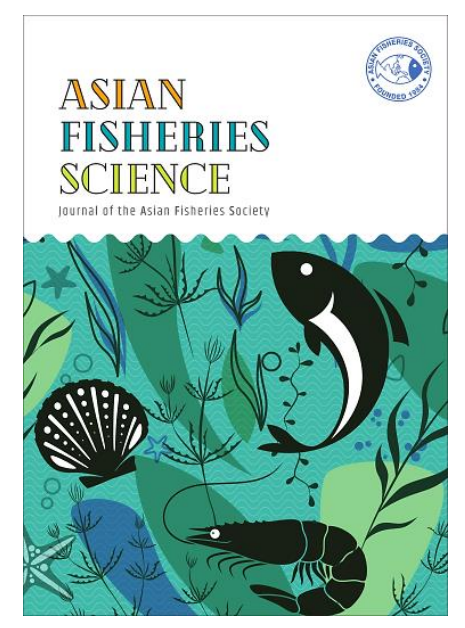

(C) Asian Fisheries Society

Published under a Creative Commons

license

E-ISSN: 2073-3720

https://doi.org/10.33997/j.afs.2021.34.4.009

\title{
Effectiveness of Adaptive Real- Time Closures for Managing Migratory Species in Data-Limited Multispecies Fisheries
}

\author{
MASAMI MUNEHARA ${ }^{1}$, METHEE KAEWNERN ${ }^{2.3}$, PAVAROT NORANARTTRAGOON $^{4}$, \\ TAKASHI FRITZ MATSUISHI ${ }^{1,2 *}$ \\ 'Faculty of Fisheries Sciences, Hokkaido University, Hakodate, Hokkaido, Japan \\ ${ }^{2}$ Global Institution for Collaborative Research and Education, Hokkaido University, Hakodate, Hokkaido, Japan \\ ${ }^{3}$ Faculty of Fisheries, Kasetsart University, Bangkok, Thailand \\ ${ }^{4}$ Department of Fisheries Thailand, Bangkok, Thailand
}

*E-mail: catm@fish.hokudai.ac.jp |Received: 24/09/2021; Accepted: 26/12/2021

\section{Abstract}

Fixed closure (FC) is a standard fisheries management tool for protecting sensitive species or species requiring conservation. However, an FC might not effectively manage migratory species because of the large uncertainties of their migration. Adaptive real-time closure (ARTC) is a tool that updates closure areas according to the latest information. This study aimed to evaluate the effectiveness of ARTC to conserve sensitive species for data-limited fisheries by a series of simulations using migration models with hotspots. In the single species simulation, the conservation ratio for the sensitive species in FC varies widely at greater migration uncertainty. In ARTC, a longer duration of a hotspot resulted in a higher conservation ratio. When the mean duration of hotspots was medium or long, the conservation ratio for the sensitive species was more than $50 \%$ in more than $99 \%$ of the simulation trials. In multispecies fisheries, a clear trade-off was observed between the conservation ratio of sensitive species and other species. ARTC was more effective than whole closure when the proportion of sensitive species was high or without closure when the proportion was low. Conditions in which ARTC was most appropriate were described for hotspot duration, increased numbers of individuals in a hotspot, and the relative value of conservation, representing the ratio of the value of conserving sensitive species to one of catching other species.

Keywords: fixed closure (FC), migration, simulation, sensitive species, sustainable fisheries

\section{Introduction}

Multispecies fisheries account for most of the world's fisheries (Halls et al., 2006). In tropical regions, biodiversity is higher than in more temperate waters, with more than 100 fish species sometimes being recorded in a particular area and season (Stobutzki et al., 2001; McClanahan and Mangi, 2004; Cohen and Foale, 2013). Fisheries closure has been proposed as an efficient way to reduce catch and fishing mortality for conserving sensitive species, i.e., species requiring conservation (Eliasen and Bichel, 2016; Woods et al., 2018; Munehara et al., 2021) by enhancing selectivity or by protecting critical habitat (Miethe et al., 2014; Ichinokawa et al., 2015). This approach has been applied widely for this purpose, even in multispecies fisheries management (Rijnsdorp et al., 2012).
A fixed closure (FC) is a standard fisheries management measure that involves decisions to close a designated area for a specified period. Once established, the decision is not changed. When the migration of the sensitive species is constant and a closed area or period is appropriately designed, it can effectively manage fish stocks. However, frequently for migratory species, there is considerable uncertainty in the migration route and timing (Breen et al., 2015), and an FC may not work well. Because migrations of the sensitive species are affected by many factors, including water temperature and currents (Punzón and Villamor, 2009; Peer and Miller, 2014; Kanamori et al., 2019), FC sometimes cannot adequately protect the sensitive species because of mismatches in the closure area and timing of migration (Dunn et al., 2016; Woods et al., 2018). 
In multispecies fisheries, it may be challenging to reduce the catch of sensitive species while maintaining the catch of other species with FC (Holmes et al., 2011; Woods et al., 2018; Watson et al., 2019). Since multispecies fisheries typically use lessselective fishing gears and exploit mixed fish stocks (Murawski et al., 1983; Murawski, 1991), FC must either expand a closed area or extend the closure period to cover uncertainties in the migration of sensitive species.

Adaptive real-time closure (ARTC) may represent a solution to the problem of FCs. This approach can adapt to uncertainties in the migration of sensitive species by using almost real-time catch data. Such management involves establishing multiple temporary closure periods (Le Quesne and Codling, 2009; Needle and Catarino, 2011) instead of one extensive, continuous closure period. In the undesirable event of a high catch of a sensitive species, an area or period at which an event occurred would be closed immediately, for example, in the Scottish cod fisheries, closure area is triggered by an upper limit on the observed cod density (Needle and Catarino, 2011). Depending on the sensitive species' distribution, ARTC would enable a high level of conservation and limit the unnecessary reduction in catch of other commercially important species. In practice, ARTC is recognised as an effective management tool in temperate waters (Needle and Catarino, 2011; Bethoney et al., 2013; Gullestad et al., 2015; Little et al., 2015).

Despite its theoretical value, the use of ARTC remains uncommon in data-limited fisheries. However, in some European countries and the USA, where appropriate datasets with a high spatiotemporal resolution exist, ARTC is more commonly used. Realtime catch data collection is costly, and establishing an effective data collection scheme for ARTC is timeconsuming process (Hobday et al., 2014). Consequently, the limited evaluation of ARTC effectiveness makes it hard to justify establishing expensive data collection schemes (Hobday and Hartmann, 2006; Dunn et al., 2011, 2016).

A hotspot is defined as an area or period in which fish are concentrated above some threshold level. The probability of meeting management goals of conserving sensitive species while maintaining the catch of other commercially important species through ARTC is affected by the duration of a hotspot and the number of fish within it (Lewison et al., 2009; Diamond et al., 2010). Presently, hotspot analysis of catch data (such as autocorrelation analysis) has been used to estimate the effectiveness of ARTC before their implementation (Dunn et al., 2011, 2014). Thus, the migration model in the present study was simulated to evaluate the effectiveness of ARTC for data-limited fisheries that incorporate species hotspots. The objectives were to evaluate the effectiveness of ARTC to conserve sensitive species with uncertain migration in multispecies fisheries and propose simple simulations as evaluation methods for fisheries without datasets.

\section{Materials and Methods}

\section{Simulation overview}

For simple representation, we assume in our simulations that individual fish move on a line at a fixed speed (Fig. 1) (Le Quesne and Codling, 2009; Watson et al., 2019). The fishing ground is defined as a fixed segment on the line. A time step is a duration in which an individual passes through a segment. The period when a fish appears in the fishing ground is defined as the appearance period. Fishing is banned during a closure period, and all fish individuals are conserved. It is assumed that all individuals in a fishing ground outside the closure period are caught.

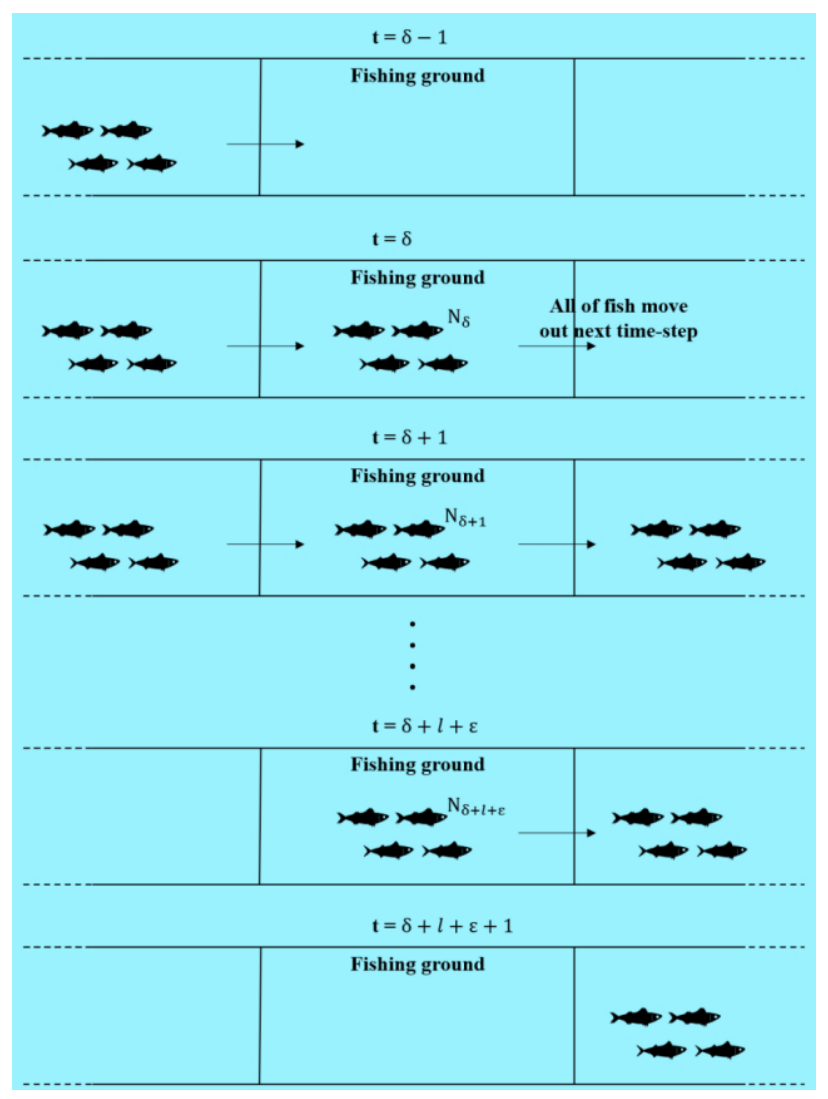

Fig. 1. Representation of space in simulations, with the fishing ground represented by a segment on the line. The fishing ground is defined as a fixed segment on the line. A time step is a duration in which an individual passes through a segment. $\delta$ is the timing when fish appears in the fishing ground. $l$ is the length of the appearance period, and $\varepsilon$ is the uncertainty of the length of the appearance period.

Two simulations were conducted. First, single sensitive species were distributed, and the conservation ratio (defined in section Effectiveness index) of FC and ARTC were evaluated. Second, other species occur in the appearance period of the sensitive species, and the effectiveness of the ARTC was examined. 


\section{Simulation model}

The number of individuals of a sensitive species in a fishing ground in a given time step $t\left(N_{t}\right)$ is described as follows:

$N_{t} \sim\left\{\begin{array}{c}n \\ n(1+\theta) \\ 0\end{array}\right.$

for $\delta^{\prime} \leq \mathrm{t}<\varepsilon_{i}, \varepsilon_{i}+\eta_{i}<\mathrm{t}<\varepsilon_{i+1}, \varepsilon_{p}+\eta_{p}<t \leq l_{a}+\delta^{\prime}$

for $\varepsilon_{i} \leq \mathrm{t} \leq \varepsilon_{i}+\eta_{i}$

for $t<\delta^{\prime}$ or $l_{a}+\delta^{\prime}<t$

where $l_{a}$ is the length of appearance period in the fishing ground, $n$ is the number of individuals in a time-step outside the hotspot, $\theta$ is the rate of increase in the number of individuals in a hotspot compared with outside hotspots, $\delta$ is a random number from a truncated normal distribution to describe uncertainties at the beginning of an appearance period, $\varepsilon_{i}$ and $\eta_{i}$ are the beginning time and duration for the ith hotspot, and $p$ is the number of hotspots (Fig. 2). For examining the effect of uncertainties arising from movement, the simulations assume a short period, and other interannual biological processes such as recruitment are ignored. In this model, $N_{t}$ is adjusted to fulfil the condition below:

$$
\sum_{\delta^{\prime}}^{l+\delta^{\prime}} N_{t}=N
$$

where $N$ is the total number of sensitive species.

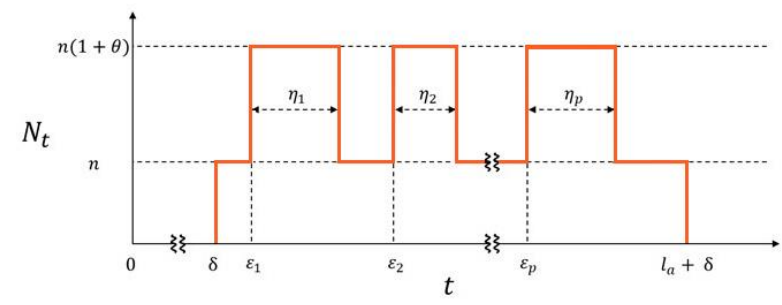

Fig. 2. Dynamics of the number of individuals in a simulation, where $\theta$ is the rate of increase in number of individuals, $\varepsilon_{1}$ and $\eta_{i}$ are the beginning time and duration for the ith hotspot, $p$ is the number of hotspots.

The beginning of the appearance period $\delta$ is determined by using the truncated normal distribution for describing migration uncertainty:

$\delta \sim\left\{\frac{1}{m} N\left(0,\left(\frac{l \times \alpha}{z(0.025)}\right)^{2}\right) \quad(-l \times \alpha \leq \delta \leq l \times \alpha)\right.$

where $m$ is the parameter for truncation $(0.95), z$ is the normal equivalent deviation (i.e., $z(0.025) \cong 1.96$ ), and $\alpha$ is the arbitrary scale of uncertainty (Table 1); and $\delta$ is converted to an integer by rounding off the value as follows:

$\delta^{\prime}=$ floor $(\delta+0.5)$

The duration for a hotspot $\eta_{i}$ follows a poison distribution:

$\eta_{i} \sim P\left(l_{h}\right)$

where three patterns for the mean duration for a hotspot $I_{\eta}$ are tested in three cases such as short (2 days), medium (1 week), long (2 weeks) when the appearance period is one month (30 days)(Table 1).

The beginning of a hotspot $\varepsilon_{i}$ is randomly changed by each iteration. It is assumed that hotspots do not occur continuously. The effect of the parameter sets (Table 1) on the results are discussed later. We performed 1000 iterations to consider the uncertainty of the timing of migration.

In the second simulation, the number of individuals of other species in the fishing ground in a given time step $t\left(M_{t}\right)$ is assumed to be constant:

$M_{t} \sim \begin{cases}\frac{M}{l_{a}} & \text { for } 0 \leq t \leq l_{a}+\delta^{\prime} \\ 0 & \text { for } \delta^{\prime}<0 \text { or } l_{a}+\delta^{\prime}<t\end{cases}$

$\sum_{\delta^{\prime}}^{l+\delta^{\prime}} M_{t}=M$

where $M$ is the total number of other species, and $M$ equals $N$ for simulation simplicity.

Table 1. Parameter set for simulations; I is the duration of the appearance period without uncertainties, $m$ is the parameter for truncation, and $\alpha_{\delta}$ is the scale of uncertainties for the beginning and length of the appearance period.

\begin{tabular}{ll}
\hline Parameter & Value \\
\hline$l_{a}$ & 30 \\
$m$ & 0.95 \\
$\alpha$ & $0,0.1,0.2,0.3,0.4,0.5,0.6,0.7,0.8$ \\
$l_{h}$ & 2 (short), 7 (medium), 14 (long) \\
$\theta$ & $0,0.5,1.0,1.5,2.0,2.5,3.0,3.5,4.0,4.5,5.0$ \\
\hline
\end{tabular}

\section{Effectiveness index}

In the first simulation (single species), the effectiveness was determined from the conservation ratio of the sensitive species $\left(\omega_{u}\right)$ was calculated as follows: 
$\omega_{\mathrm{u}}=\sum_{t=\delta^{\prime}}^{l_{a}+\delta^{\prime}} \frac{x_{t} N_{t}}{N} x_{t} \in\{0,1\} \forall t$.

This value was multiplied by a binomial variable $x_{t}$ (i.e. 0 or 1), representing whether the time step is included in the closure period. In these simulations, the duration of the FC is $t=0$ to $I_{a}$ in the ARTC, when a hotspot is outside the closure period, the closure period begins in the next time step and continues for $\lambda$ time-steps. As a default, $\lambda=I_{\eta}$.

In the second simulation, the effectiveness index $(\varphi)$ was calculated using two aspects of the conservation ratio of the sensitive species $\left(\omega_{u}\right)$ and catch ratio of other species $\left(\omega_{0}\right)$.

$\omega_{\mathrm{o}}=\sum_{t=\delta^{\prime}}^{l_{a}+\delta^{\prime}} \frac{\left(1-x_{t}\right) M_{t}}{M} x_{t} \in\{0,1\} \forall t$.

$\varphi=\sum_{t=\delta^{\prime}}^{l_{a}+\delta^{\prime}}\left(\rho \omega_{2_{\mathrm{c}}}+\omega_{2_{\mathrm{o}}}\right)$

where $\rho$ was the relative value of sensitive species when the value of the other species was 1.

The whole closure was defined as the closure through the entire appearance period. The effectiveness of the ARTC was expressed by the differences of the effectiveness index of ARTC, whole closure ( $\varphi_{\text {ARTC }}-$ $\left.\varphi_{\mathrm{wC}}\right)$, and without closure $\left(\varphi_{\text {ARTC }}-\varphi_{\text {woc }}\right)$. We defined ARTC to be effective when both of $\varphi_{\text {ARTC }}-\varphi_{\mathrm{wc}}$ and $\varphi_{\text {ARTC }}$ - $\varphi_{\text {woc }}$ have positive values, and detected the condition of $\rho$ and $\theta$ in which ARTC is effective.

\section{Results}

In the first simulation, the conservation ratio for sensitive species in FC $\omega_{\mathrm{u}_{\mathrm{FC}}}$ varies widely at greater uncertainty $\alpha$ (Fig. 3). When $\alpha=0.8$, the frequency of $\omega_{\mathrm{u}_{\mathrm{FC}}}>90 \%$ was 171 out of 1000 iterations. However, at $\omega_{\mathrm{u}_{\mathrm{FC}}}<30 \%$, this falls to 36 out of 1000 iterations. In ARTC, a longer duration of a hotspot $I_{\eta}$ resulted in a higher conservation ratio $\omega_{\mathrm{u}_{\mathrm{ARTC}}}($ Fig. 4$)$. When the mean duration of a hotspot was short $\left(I_{\eta}=2\right), \omega_{\mathrm{u}_{\mathrm{ARTC}}}$ $<50 \%$ was observed in more than half of the iterations at even the largest number of individuals in a hotspot $(\theta=5.0)$. Conversely, when the mean duration of hotspots was medium or long $\left(I_{\eta}=7\right.$ and $14-$ time steps), the frequency of $\omega_{\mathrm{u}_{\mathrm{ARTC}}}<50 \%$ was 11 and 3 out of 1000 iterations, respectively.

In the second simulation, a clear trade-off was observed between the conservation ratio of sensitive species $\omega_{\mathrm{u}}$ and other species $\omega_{0}$ (Fig. 5). When $\omega_{0}$ was the same in every $I_{\eta,} \omega_{u}$ tends to be highest with long $I_{\eta}$. The range of $\omega_{\mathrm{u}}$ was narrower than $\omega_{0}$ across $I_{\eta}$ at $\theta$

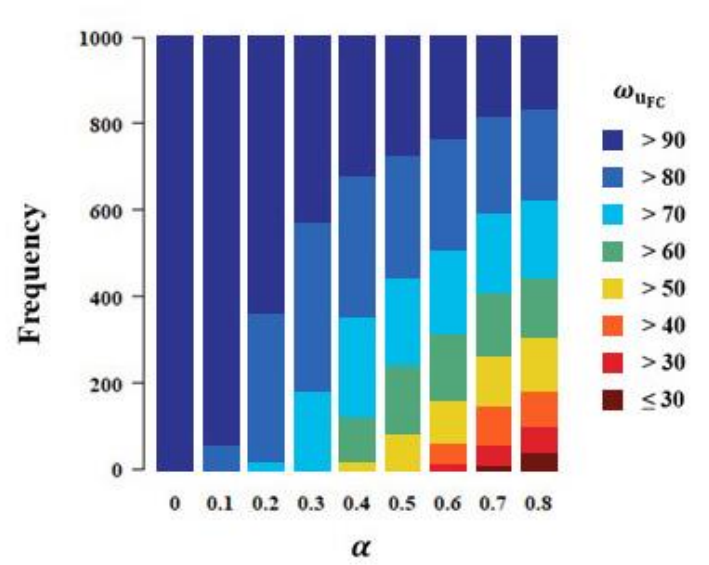

Fig. 3. Frequency of each conservation ratio $\omega_{\mathrm{u}_{\mathrm{FC}} ; \alpha \text { is a }}$ scale of uncertainty at the beginning of the appearance period, when a fish first appears in the fishing ground.

$=2.5$ or 5.0 . Multiple peaks were observed in density distribution for $\omega_{u}$ or $\omega_{0}$, mainly when $I_{\eta}$ is medium.

Differences in the effectiveness index $\varphi$ between ARTC and whole closure $\left(\varphi_{\text {ARTC }}-\varphi_{\mathrm{wc}}\right)$, and one between ARTC and without closure $\left(\varphi_{\mathrm{ARTC}}-\varphi_{\mathrm{woc}}\right)$, are presented in Figure 6. ARTC was more effective than whole closure when $\rho$ was high, and without closure when $\rho$ was low. The combination of $\rho$ and $\theta$ in which $\varphi_{\text {ARTC }}$ was higher than $\varphi_{\text {wc }}$ and $\varphi_{\text {woc }}$ was identified (Fig. 7). The area where ARTC was more effective than whole closures or without closure increases as $I_{\eta}$ increases.

\section{Discussion}

The present study investigated the effectiveness of ARTC for migratory species management in multispecies fisheries. Adaptive real-time closure was demonstrated to be an effective management tool for maximising benefits from conserving sensitive species and catching others. Adaptive realtime closure works well, especially when the differences in value between conserving sensitive species and catching other species are small (Fig. 7).

\section{Comparison of FC and ARTC in single- species management}

The main factor impacting the conservation ratio of FC and ARTC in single-species fisheries differs. The conservation ratio of $\mathrm{FC}$ is affected mainly by uncertainty at the beginning of an appearance period. In contrast, the fluctuation in the number of individuals in the appearance period did not significantly impact the FC. On the contrary, the latter is a more critical factor for ARTC (Dunn et al., 2016; Woods et al., 2018). Thus, the conservation ratio of FC and ARTC is evaluated by changing $\alpha$ and $\theta_{i}$ respectively.

When the $\alpha$ value was small, FC had a higher conservation ratio than ARTC for any $\theta$ and $I_{\eta}$. From 

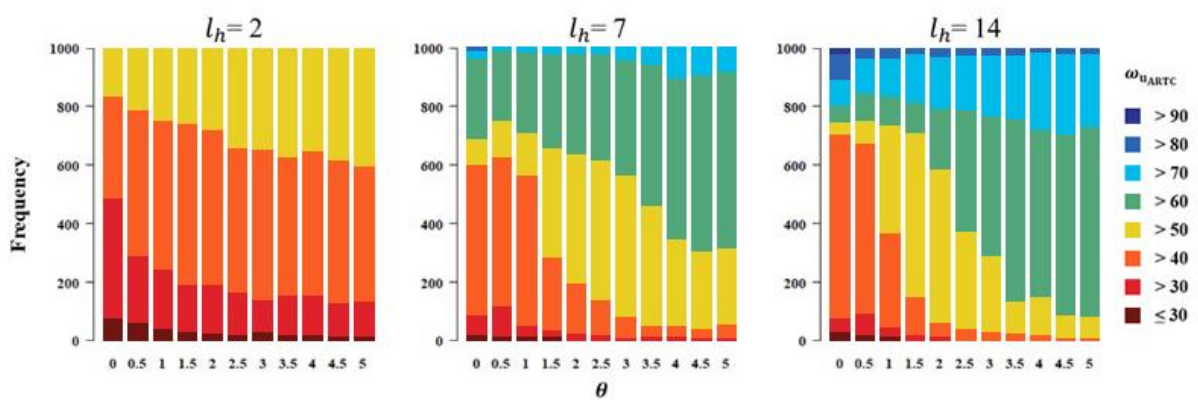

Fig. 4. Frequency of each conservation ratio $\left(\omega_{\mathrm{u}_{\mathrm{ARTC}}}\right)_{i} I_{\eta}$ is the mean duration of a hotspot period, $\theta$ is the increased number of individuals in a hotspot.
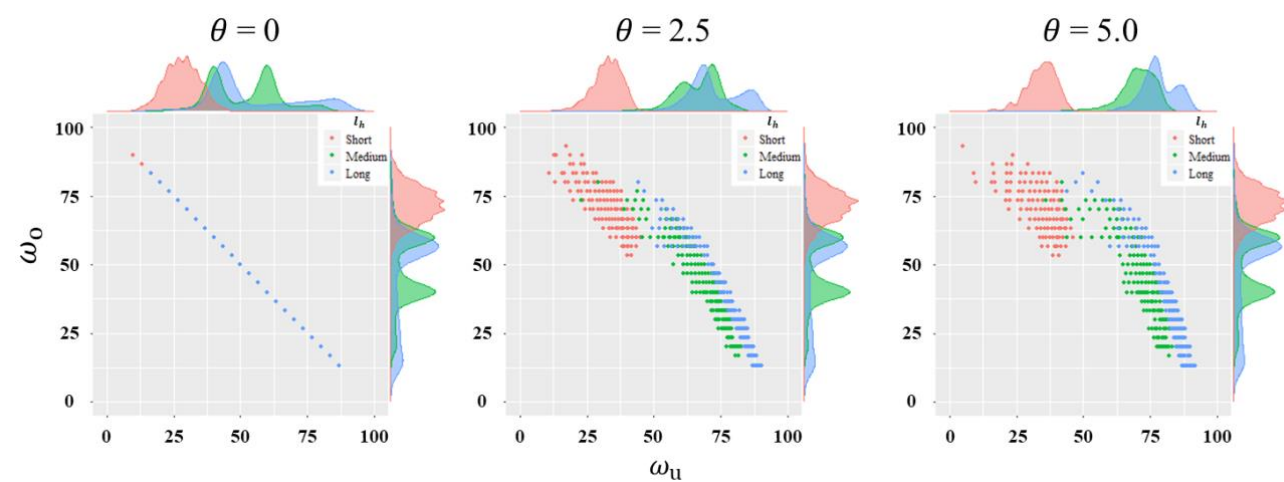

Fig. 5. Adaptive real-time closure scatter plot for conservation ratio $\left(\omega_{u}\right)$ and catch ratio $\left(\omega_{0}\right)_{i} I_{\eta}$ is the mean length of a hotspot period, short (red), medium (green), and long (blue). The value of $\theta$ is the increased number of individuals in a hotspot. The area graph on the top and right side of the graph plain shows the distribution of $\omega_{\mathrm{u}}$ and $\omega_{0}$.
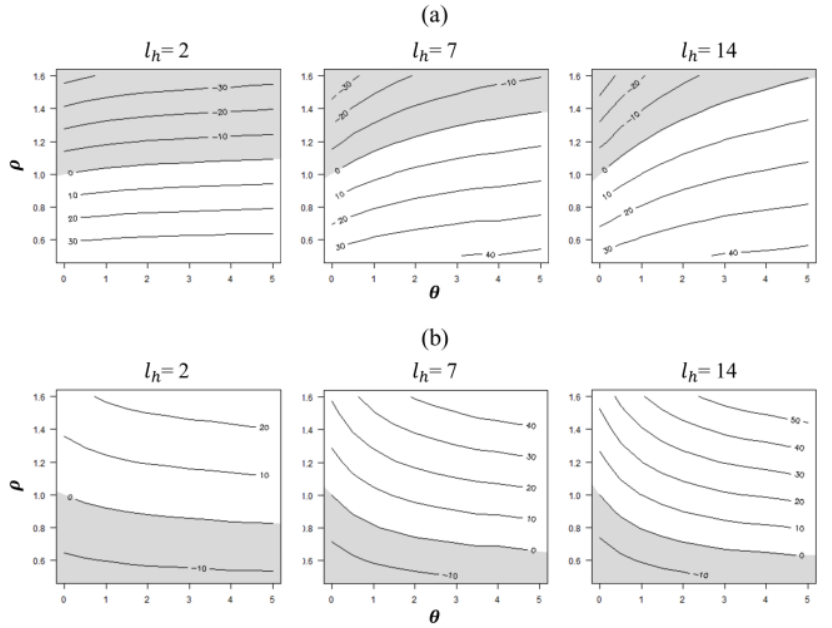

Fig. 6. The difference in effectiveness index $\varphi$ between (a) adaptive real-time closure (ARTC) and whole closure $\left(\varphi_{\mathrm{ARTC}}-\varphi_{\mathrm{wc}}\right)$, and (b) ARTC and no closure $\left(\varphi_{\text {ARTC }}-\varphi_{\text {woc }}\right)$. Values are negative in the grey zone; $I_{\eta}$ is the mean hotspot duration, $\theta$ is the increased number of individuals in a hotspot, and $\rho$ is the relative value, which is the ratio of the value of conserving sensitive species to one of catching other species.
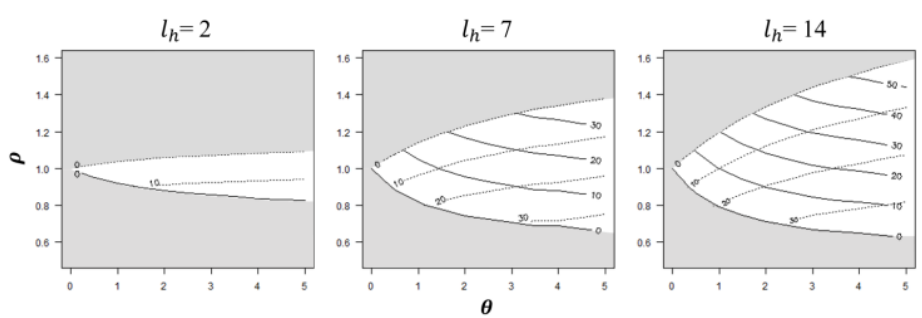

Fig. 7. The combination of $\left(\varphi_{\text {ARTC }}-\varphi_{\text {vc }}\right)\left[\right.$ dotted line] and $\left(\varphi_{\text {ARTC }}-\varphi_{\text {woc }}\right)$ [solid line]. Values are negative in the grey zone; $\theta$ is the increased number of individuals in a hotspot, and $\rho$ is the relative value (values are negative in the grey zone), which is the ratio of the value of conserving sensitive species to one of catching other species. 
these results, FC would be the first choice if the sole objective was to conserve a sensitive species with little uncertainty in its migration behaviour. If there was considerable uncertainty in a species' migratory behaviour, the conservation ratio of an FC fluctuates widely (Fig. 3). In this case, ARTC represents an alternative method to conserve a species, depending on the duration of the hotspot and the number of individuals within it.

\section{ARTC in multispecies fisheries}

The conservation ratio of sensitive species $\omega_{u}$ and catch ratio of other species $\omega_{0}$ are evaluated to appraise the effectiveness of ARTC in multispecies fisheries. The range of $\omega_{0}$ is wider than that of $\omega_{11}$ and multiple peaks are apparent in the density distribution for $\omega_{1}$ and $\omega_{0}$ (Fig. 5). In this simulation, the duration of the ARTC is determined to be equal to the mean hotspot duration $I_{\eta ;}$ ARTC commences in the next time step in which a sensitive species is caught within a hotspot. Thus, in ARTC, if actual hotspot duration $\eta_{i}$ is longer than $I_{\eta}$, the next closure period begins immediately after the previous closure period finishes, causing multiple peaks in density distribution.

The optimum closure period of ARTC will change with management objectives (Little et al., 2015). Although estimating the optimum closure duration was not our main objective, we performed a further simulation that involved changing the ARTC closure duration to estimate the period required to maximise $\varphi_{\text {ARTC }}$ (Table 2). The $\omega_{u}$ and $\omega_{0}$ scatter plots for this simulation are presented in Figure 8. When $I_{\eta}$ was medium, the closure duration was shortened, the points shift from the upper right to the bottom left, and the range in $\omega_{0}$ decreases further than in Fig. 5. Conversely, when $I_{\eta}$ was long, the closure duration was extended, and the points shift to enhance $\omega_{l}$.

Table 2. The ARTC closure period required to maximise $\varphi_{A R T C}$. Default values for $I_{\eta}$ are 2 (short), 7 (medium) and 14 (long).

\begin{tabular}{llll}
\hline$l_{h}$ & $\theta=0$ & $\theta=2.5$ & $\theta=5.0$ \\
\hline Short & 1 & 1 & 1 \\
Medium & 1 & 4 & 16 \\
Long & 1 & 3 & 15 \\
\hline
\end{tabular}

The present study identified the combination of the relative value of sensitive species $\rho$ and the number of individuals in a hotspot $\theta$ for which an ARTC approach is most effective. When $\varphi_{\text {ARTC }}-\varphi_{\text {woc }}$ was negative, it was more effective to close during the entire appearance period of sensitive species than ARTC. Conversely, fisheries closure is unsuitable when $\varphi_{\text {ARTC }}-\varphi_{w c}$ is negative. These results enable estimation of the value of ARTC to facilitate effective management because there is a compromise between the benefits of enhanced conservation of species provided by an ARTC and the cost of its introduction.

\section{Model assumptions}

There has been considerable development and evaluation of spatial modelling techniques to improve fisheries management in recent years (Goethel et al., 2011; Berger et al., 2017; Punt, 2019). Several models have been developed to evaluate fisheries closure by simulation, such as individual-based models (Moustakas and Silvert, 2011; Cornejo-Donoso et al., 2017), diffusion-based models (Le Quesne and Codling, 2009; Watson et al., 2019), and preferred habitat models (Ono et al., 2013). The most crucial factor affecting ARTC is hotspots in species distribution (Little et al., 2015; Eliasen and Bichel, 2016). If hotspots are incorporated into these models, many parameters would be needed, and they would become complicated. Dunn et al. (2016) simulated the effects of ARTC in the management of Atlantic cod fisheries by analysing autocorrelation for past highresolution fishing data. However, no previous study has evaluated the effectiveness of ARTC in simulations, and thus, this study establishes a new simple model that can assess ARTC even in datalimited fisheries. From this result, if the hotspot duration and the increased number of individuals in a hotspot compared with outside hotspots are roughly estimated, ARTC can be evaluated without a highresolution dataset.

The present model assumes that individuals move along a line at a fixed speed and that the fishing ground represents a segment on this line. The present study simulates the dynamics of several fish in a particular fishing ground. The hotspot duration and the relative value of conserving sensitive species are focused on as the factors affecting the effectiveness of ARTC. Adaptive real-time closure
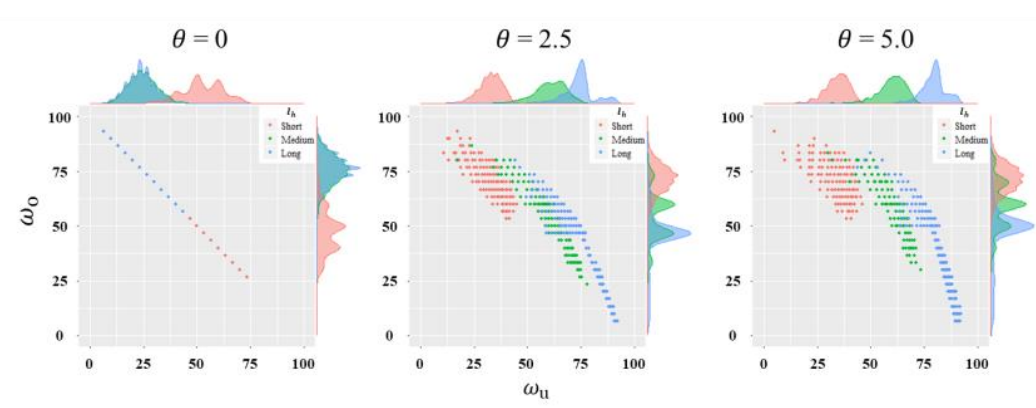

Fig. 8. Adaptive real-time closure scatter plot for conservation ratio $\left(\omega_{u}\right)$ and catch ratio $\left(\omega_{0}\right)$, when closure periods are applied from Table 2; $I_{\eta}$ represents the mean hotspot duration: short (red), medium (green), and long (blue). The value of $\theta$ is the increased number of individuals in a hotspot. 
prevents the continuous occurrence of adverse events, such as catching in hotspots of sensitive species after their initial occurrence (Gaines et al., 2010; Dunn et al., 2011). Thus, hotspot duration was incorporated into the simulation as a variable, in addition to a coefficient for an increased number of individuals within a hotspot.

The relative value is the conservation value of a sensitive species divided by the value of catching other species. In multispecies fisheries, it is necessary to consider the conservation of sensitive species and the economic loss resulting from the reduced catch of other species. Thus, the relative economic value was incorporated into our simulation to evaluate these two aspects. A conservation value can be calculated by estimating the financial loss resulting from the catch of a sensitive species. For example, if a juvenile fish is considered a sensitive species, catching an immature juvenile contributes to decreased recruitment and loss of future benefit (Noranarttragoon, 2007; Najmudeen and Sathiadhas, 2008). Thus, the value of juvenile fish can be estimated by calculating the economic loss resulting from failing to conserve it.

\section{Conclusion}

The present study evaluated the effectiveness of adaptive real-time closure (ARTC) with simple movement models. While spatial-temporal population dynamic modelling has increased rapidly over the last two decades, no previous model has described hotspots or used them to evaluate the effectiveness of ARTC. The simulation of ARTC in the present study represents a step towards sustainable management of migratory species in multispecies fisheries while contending with data-limited conditions. Future improvements to movement assumptions of the fish and spatial-temporal data availability will enable the application of more sophisticated simulation models. Although the practical use of ARTC remains limited, the results of these simulations may be used as a reference to expanding their use in multispecies and data-limited fisheries.

\section{Acknowledgements}

Gl-Core, Hokkaido University, supports this research. We also thank Steve O'Shea, PhD, from Edanz Group (https://en-author-services.edanzgroup.com/ac) for editing a draft of this manuscript.

Conflict of interest: The authors declare that they have no conflict of interest.

\section{References}

Berger, A.M., Goethel, D.R., Lynch, P.D., Terrance Quinn, Mormede, S., Mckenzie, J., Dunn, A. 2017. Space oddity: The mission for spatial integration 1. Canadian Journal of Fisheries and Aquatic Sciences 74:1698-1716. https://doi.org/10.1139/cjfas-2017-0150
Bethoney, N.D., Schondelmeier, B.P., Stokesbury, K.D.E., Hoffman, W.S. 2013. Developing a fine scale system to address river herring (Alosa pseudoharengus, A. aestivalis) and American shad (A. sapidissima) bycatch in the US Northwest Atlantic mid-water trawl fishery. Fisheries Research 141:79-87. https://doi.org/10.1016 /j.fishres.2012.09.003

Breen, P., Posen, P., Righton, D. 2015. Temperate marine protected areas and highly mobile fish: A review. Ocean and Coastal Management. https://doi.org/10.1016/j.ocecoaman.2014.12.021

Cohen, P.J., Foale, S.J. 2013. Sustaining small-scale fisheries with periodically harvested marine reserves. Marine Policy 37:278-287. https://doi.org/10.1016/j.marpol.2012.05.010

Cornejo-Donoso, J., Einarsson, B., Birnir, B., Gaines, S.D. 2017. Effects of fish movement assumptions on the design of a marine protected area to protect an overfished stock. PLoS ONE 12. https://doi.org /10.1371/journal.pone.0186309

Diamond, S.L., Kleisner, K.M., Duursma, D.E., Wang, Y. 2010. Designing marine reserves to reduce bycatch of mobile species: a case study using juvenile red snapper (Lutjanus campechanus). Canadian Journal of Fisheries and Aquatic Sciences 67:1335-1349. https://doi.org/10.1139/F10-044

Dunn, D.C., Boustany, A.M., Halpin, P.N. 2011. Spatio-temporal management of fisheries to reduce by-catch and increase fishing selectivity. Fish and Fisheries 12:110-119. https://doi.org/10.1111 /j.1467-2979.2010.00388.x

Dunn, D.C., Boustany, A.M., Roberts, J.J., Brazer, E., Sanderson, M., Gardner, B., Halpin, P.N. 2014. Empirical move-on rules to inform fishing strategies: A New England case study. Fish and Fisheries 15:359-375. https://doi.org/10.1111/faf.12019

Dunn, D.C., Maxwell, S.M., Boustany, A.M., Halpin, P.N. 2016. Dynamic ocean management increases the efficiency and efficacy of fisheries management. Proceedings of the National Academy of Sciences 113:668-673. https://doi.org/10.1073/pnas.1513626113

Eliasen, S.0., Bichel, N. 2016. Fishers sharing real-time information about "bad" fishing locations. A tool for quota optimisation under a regime of landing obligations. Marine Policy 64:16-23. https://doi.org/10.1016/j.marpol.2015.11.007

Gaines, S.D., White, C., Carr, M.H., Palumbi, S.R. 2010. Designing marine reserve networks for both conservation and fisheries management. Proceedings of the National Academy of Sciences 107:18286-18293. https://doi.org/10.1073/pnas.0906473107

Goethel, D.R., Quinn, T.J., Cadrin, S.X. 2011. Incorporating spatial structure in stock assessment: Movement modeling in marine fish population dynamics. Reviews in Fisheries Science \& Aquaculture 19:119-136. https://doi.org/10.1080/10641262.2011.557451

Gullestad, P., Blom, G., Bakke, G., Bogstad, B. 2015. The "Discard Ban Package": Experiences in efforts to improve the exploitation patterns in Norwegian fisheries. Marine Policy 54:1-9. https://doi.org /10.1016/j.marpol.2014.09.025

Halls, A.S., Welcomme, R.L., Burn, RW 2006. The relationship between multispecies catch and effort: Among fishery comparisons. Fisheries Research 77:78-83. https://doi.org/10.1016/j.fishres .2005.08.005

Hobday, A.J., Hartmann, K. 2006. Near real-time spatial management based on habitat predictions for a longline bycatch species. Fisheries Management and Ecology 13:365-380. https://doi.org /10.1111/j.1365-2400.2006.00515.x

Hobday, A.J., Maxwell, S.M., Forgie, J., McDonald, J., Darby, M., Seto, K. Bailey, H., Bograd, S.J., Briscoe, D.K., Costa, D.P., Crowder, L.B. Dunn, D.C., Fossette, S., Halpin, P.N., Hartog, J.R., Hazen, E.L., Lascelles, B.G., Lewison, R.L., Poulos, G., Powers, A. 2014. Dynamic ocean management: Integrating scientific and technological 
capacity with law, policy and management. Stanford Environmental Law Journal 33:125-165.

Holmes, S.J., Bailey, N., Campbell, N., Catarino, R., Barratt, K., Gibb, A., Fernandes, P.G. 2011. Using fishery-dependent data to inform the development and operation of a co-management initiative to reduce cod mortality and cut discards. ICES Journal of Marine Science 68:1679-1688. https://doi.org/10.1093/icesjms/fsr101

Ichinokawa, M., Okamura, H., Watanabe, C., Kawabata, A., Oozeki, Y. 2015. Effective time closures: Quantifying the conservation benefits of input control for the Pacific chub mackerel fishery. Ecological Applications 25:1566-1584. https://doi.org/10.1890/14-1216.1

Kanamori, Y., Takasuka, A., Nishijima, S., Okamura, H. 2019. Climate change shifts the spawning ground northward and extends the spawning period of chub mackerel in the western North Pacific. Marine Ecology Progress Series 624:155-166. https://doi.org /10.3354/meps13037

Le Quesne, W.J.F., Codling, E.A. 2009. Managing mobile species with MPAs: The effects of mobility, larval dispersal, and fishing mortality on closure size. In: ICES Journal of Marine Science, Oxford Academic, pp. 122-131. https://doi.org/10.1093/icesjms/fsn202

Lewison, R.L., Soykan, C.U., Franklin, J. 2009. Mapping the bycatch seascape: multispecies and multi-scale spatial patterns of fisheries bycatch. Ecological Applications 19:920-930. https://doi.org/10.1890 /08-0623.1

Little, AS, Needle, C.L., Hilborn, R., Holland, D.S., Marshall, C.T. 2015. Real-time spatial management approaches to reduce bycatch and discards: Experiences from Europe and the United States. Fish and Fisheries 16:576-602. https://doi.org/10.1111/faf.12080

McClanahan, T.R., Mangi, S.C. 2004. Gear-based management of a tropical artisanal fishery based on species selectivity and capture size. Fisheries Management and Ecology 11:51-60. https://doi.org/10.1111/j.1365-2400.2004.00358.x

Miethe, T., Bastardie, F., von Dorrien, C., Nielsen, J.R. 2014. Impact assessment of a fisheries closure with effort and landings spatial analyses: A case study in the Western Baltic Sea. Fisheries Research 157:170-179. https://doi.org/10.1016/j.fishres.2014.04.004

Moustakas, A., Silvert, W. 2011. Spatial and temporal effects on the efficacy of marine protected areas: Implications from an individual based model. Stochastic Environmental Research and Risk Assessment 25:403-413. https://doi.org/10.1007/s00477-010-0411-2

Munehara, M., Kaewnern, M., Noranarttragoon, P., Matsuishi, T.F. 2021. Simulations of fixed closure and real-time closure to manage migratory fish species for data-limited fisheries. Ocean and Coastal Management 211. https://doi.org/10.1016/j.ocecoaman.2021.105736

Murawski, S.A. 1991. Can we manage our multispecies fisheries? Fisheries 16:5-13. https://doi.org/10.1577/1548-8446(1991)016 $<0005$ :cwmomf>2.0.co;2
Murawski, S.A., Lange, A.M., Sissenwine, M.P., Mayo, R.K. 1983. Definition and analysis of multispecies otter-trawl fisheries off the northeast coast of the United States. ICES Journal of Marine Science 41:13-27. https://doi.org/10.1093/icesjms/41.1.13

Najmudeen, T.M., Sathiadhas, R. 2008. Economic impact of juvenile fishing in a tropical multi-gear multispecies fishery. Fisheries Research 92:322-332. https://doi.org/10.1016/j.fishres.2008.02.001

Needle, C.L., Catarino, R. 2011. Evaluating the effect of real-time closures on cod targeting. ICES Journal of Marine Science 68:16471655. https://doi.org/10.1093/icesjms/fsr092

Noranarttragoon, P. 2007. An evaluation on economic loss from luring purse seine fishery in the Gulf of Thailand. Kasetsart Journal (Natural Science) 41:141-148.

Ono, K., Holland, D.S., Hilborn, R. 2013. How does species association affect mixed stock fisheries management? A comparative analysis of the effect of marine protected areas, discard bans, and individua fishing quotas. Canadian Journal of Fisheries and Aquatic Sciences 70:1792-1804. https://doi.org/10.1139/cjfas-2013-0046

Peer, A.C., Miller, T.J. 2014. Climate change, migration phenology, and fisheries management interact with unanticipated consequences. North American Journal of Fisheries Management 34:94-110. https://doi.org/10.1080/02755947.2013.847877

Punt, A.E. 2019. Spatial stock assessment methods: A viewpoint on current issues and assumptions. Fisheries Research 213:132-143. https://doi.org/10.1016/j.fishres.2019.01.014

Punzón, A., Villamor, B. 2009. Does the timing of the spawning migration change for the southern component of the Northeast Atlantic Mackerel (Scomber scombrus, L. 1758)? An approximation using fishery analyses. Continental Shelf Research 29:1195-1204. https://doi.org/10.1016/j.csr.2008.12.024

Rijnsdorp, A.D., Van Overzee, H.M.J., Poos, J.J. 2012. Ecological and economic trade-offs in the management of mixed fisheries: A case study of spawning closures in flatfish fisheries. Marine Ecology Progress Series 447:179-194. https://doi.org/10.3354/meps09519

Stobutzki, I.C., Miller, M.J., Jones, P., Salini, J.P. 2001. Bycatch diversity and variation in a tropical Australian penaeid fishery; the implications for monitoring. Fisheries Research 53:283-301. https://doi.org/10.1016/S0165-7836(00)00273-3

Watson, F.M., Hepburn, L.J., Cameron, T., Le Quesne, W.J.F., Codling, E.A. 2019. Relative mobility determines the efficacy of MPAs in a two species mixed fishery with conflicting management objectives. Fisheries Research 219. https://doi.org/10.1016/j.fishres.2019.105334

Woods, P.J., Elvarsson, B. pór, Sigurdsson, T., Stefánsson, G. 2018. Evaluating the effectiveness of real-time closures for reducing susceptibility of small fish to capture. ICES Journal of Marine Science 75:298-308. https://doi.org/10.1093/icesjms/fsx152 